\title{
DESIGN, FABRICATION AND RF MEASUREMENT OF A W-BAND ACCELERATING STRUCTURE
}

\author{
R. Merte, H. Henke, R. Apel \\ Technische Universität Berlin, EN-2, Einsteinufer 17, D-10587 Berlin, Germany
}

\begin{abstract}
The paper presents a design of a W-band planar accelerating structure (muffin tin) [1]. The structure is a $2 \pi / 3$-mode traveling wave, constant impedance structure with an operating frequency of $91.392 \mathrm{GHz}$. The design includes a new power coupler and a cavity geometry optimized for high shunt impedance. A 7-cell cold test prototype has been fabricated by wire electro discharge machining. Measurements were performed with a scalar network analyzer for both Sparameters and bead pull measurements. The RF parameters and measured results are presented and compared with the numerical results obtained by GdfidL [3] simulations.
\end{abstract}

\section{INTRODUCTION}

Very high frequency accelerating structures find more and more interest. They have the potential for very high gradient application due to a higher breakdown field and a better pulsed heating behavior. But they can also be a perfect match to particular applications such as medical accelerators. Or, they may even be a must as in space applications. On the other hand, very high frequencies, say above 60 to $80 \mathrm{GHz}$, require planar geometries for fabricational reasons. While planar geometries and the necessary technologies have to be developed, this avenue may lead to completely new concepts as for instance integrated modules, where the accelerating structure, the power sources and the focusing elements are produced on a common support.

Modern micro fabrication has developed, at least, two technologies which meet the requirements for accelerating structures: wire electro-discharge-machining (WEDM) and X-ray lithography (LIGA). Both technologies allow fabricational tolerances in the micrometer or even submicrometer range and surface roughness in the order of 0.1 micrometer, and they have relative advantages and disadvantages. For WEDM the structure must be constructed in layers and brazed or diffusion bonded together and the process is not well suited for mass production. LIGA is first of all expensive since it requires an $\mathrm{X}$-ray mask which typically must be manufactured in several steps, a synchrotron radiation beam line for a many-hour exposure and facilities for developing and electroforming. It should, however, be much cheaper when producing large numbers because molding can be used. WEDM is intensely pursued at SLAC [4]. In Berlin our main focus will be on LIGA which is already available at BESSY I and where a full production line is going to be build up at BESSY II. Nevertheless, our first structure was WEDM'ed, partly for financial reasons but also because we could profit from the experience SLAC has made and use the same manufacturer.

\section{MECHANICAL DESIGN AND FABRICATION}

The first design for the structure design was presented in [2]. The prototype is a 7-cell structure suited for wire EDM. Therefore special limitations were taken into account.

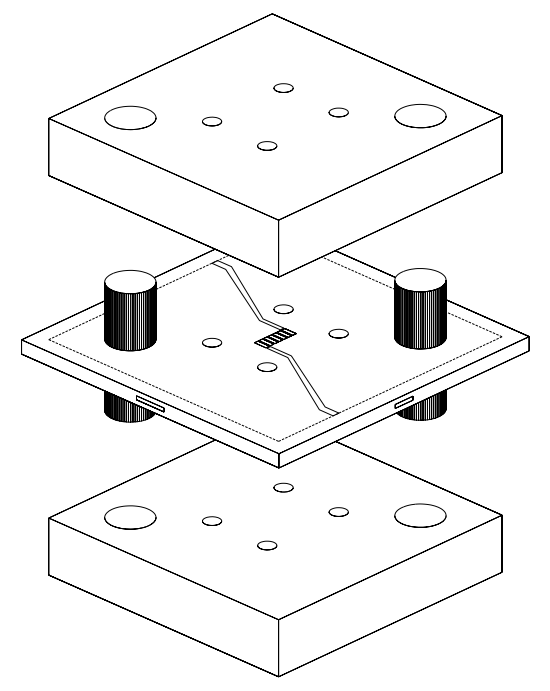

Figure 1. Explosion view of the cold test model.

Figure 1 shows the explosion view of the cold test model. The top and the bottom plates have the function of a mechanical support and represent the plates of the cavity's ground. The middle sheet is the structure with cavity section, beam pipe, pumping slots, input/output coupler plus detoured wave guides, integrated taper and holes for screws and alignment pins. The power couplers are planar and input and output are from both sides, left and right. The dashed line indicates a sacrificial region, which has been cut away after mounting the structure together. It serves the stabilization during machining. The thickness of this sheet is $2 b=2.54 \mathrm{~mm}$. The design allows two different kinds of bead pull measurements: the conventional longitudinal pull through the beam pipe and a pull with a transverse dielectric fiber, positioned and moved in the pumping slots. The 
connected WR-10 flange is as large as half the structure and overlaps the pumping slots which are needed for the transverse fiber bead pull measurement. Therefore it was necessary to detour the wave guide from the input and output coupling cells to the exterior boundary of the structure. Further the gap size of the first and last cells $g_{1}=0.864 \mathrm{~mm}$, are smaller than the size of a WR-10 wave guide $w g=1.27$ $\mathrm{mm}$, so that tapering in this direction was necessary. The transition from the coupling cells to the outer WR-10 flange via the integrated taper is smooth and optimized for minimal reflection.

Figure 2 shows a typical planar cavity in different views. The iris thickness has been fixed to $t=0.23 \mathrm{~mm}$. The length of one cavity in beam direction, the gap, results from the condition that we chose the $2 \pi / 3$-mode with $g=\lambda / 3-t=0.864 \mathrm{~mm}$. The aperture for the beam influences bandwidth, shunt impedance and wakefields. A relatively large aperture to wavelength ratio of $a / \lambda=0.16$ was chosen, which results in an aperture of $2 a=1.05 \mathrm{~mm}$. The width of the inner cavities which adjust the frequency is $w=2.363 \mathrm{~mm}$. The depth is $2 b=2.54 \mathrm{~mm}$ which is the size of the standard WR-10 wave guide and, therefore, does not require tapering in this direction.

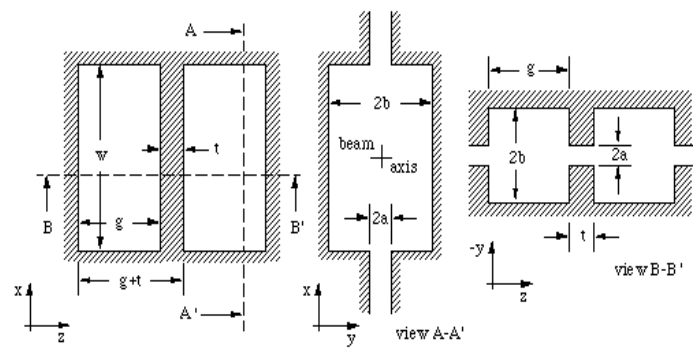

Figure 2. Typical cavity, a) top view, b) cross section and c) longitudinal cut.

The input and output couplers of the prototype have been matched with a cut coupling iris. The terminating coupling cells have a width of $w_{1}=1.89 \mathrm{~mm}$ and the coupling iris has an aperture of $a_{1}=0.79 \mathrm{~mm}$. A disadvantage is the different depth $a_{1}$ in case of a fabrication with LIGA.

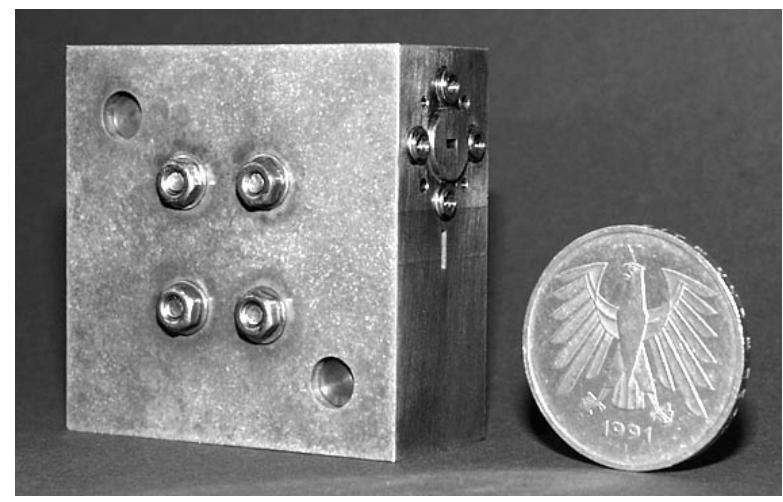

Figure 3. Prototype with RF-flange and bolts.
Figure 3 shows the final structure bolted together, which was fabricated by Ron Witherspoon, Inc., Campbell, California.

\section{RF DESIGN AND NUMERICAL SIMULATION}

As mentioned above the cold test model is a traveling wave constant impedance structure. It is designed for the $2 \pi / 3$-mode. The operating frequency is $91.392 \mathrm{GHz}, 32$ times the SLAC frequency $(2.856 \mathrm{GHz})$, and corresponds to a wavelength of $\lambda=3.283 \mathrm{~mm}$. The period length $p$ follows therefore with $p=\lambda / 3=1.094 \mathrm{~mm}$. The basic RF parameters are determined numerically with our code GdfidL [3] and listed in table 1. The cavity geometry is designed for an optimized shunt impedance. For the determination of the maximum length $L_{\max }$ of the structure, we used a $\tau$ of $0.8(\tau=\alpha l)$, resulting in a maximal number of accelerating cells of $N_{\max }=178$.

$$
\begin{gathered}
r / Q_{0}=81.6 \mathrm{k} \Omega / \mathrm{m} \\
Q_{0}=2490, r=200 \mathrm{M} \Omega / \mathrm{m} \\
v_{g} / c_{0}=9.4 \% \\
\alpha=4.1 \mathrm{~m}^{-1} \\
\rightarrow \tau=0.8 \Rightarrow L_{\text {max }}=19.5 \mathrm{~cm} \Rightarrow N_{\text {max }}=178
\end{gathered}
$$

Table 1: Basic RF parameters.

The results of the numerical simulation in the time domain of the entire structure of the reflection and transmission coefficient, computed with GdfidL, are shown in figure 4.

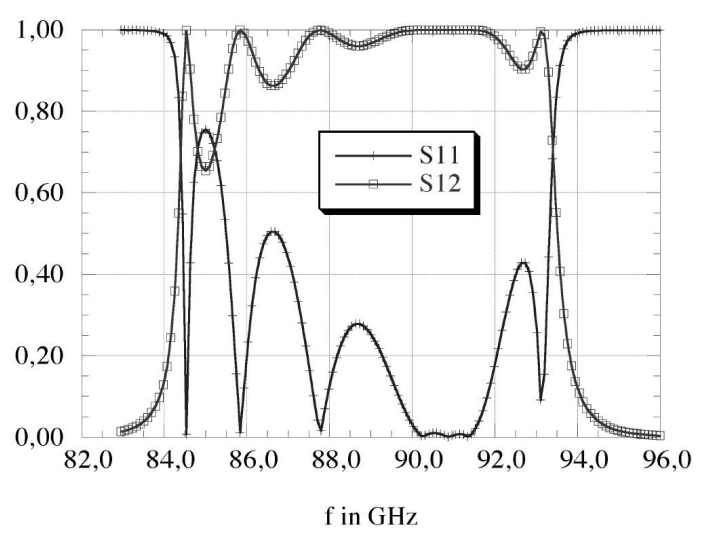

Figure 4. Reflection and transmission coefficient, GdfidL [3] simulation.

\section{RF-MEASUREMENT}

In the following a relatively cheap possibility to make transmission and reflection measurements as well as bead pull 
measurement with a scalar measurement system in the Wband $(75-110 \mathrm{GHz})$, are shown. Figure 5 shows the block diagram of the scalar measurement system for transmission and reflection measurements and figure 6 the set up for bead pull measurements.

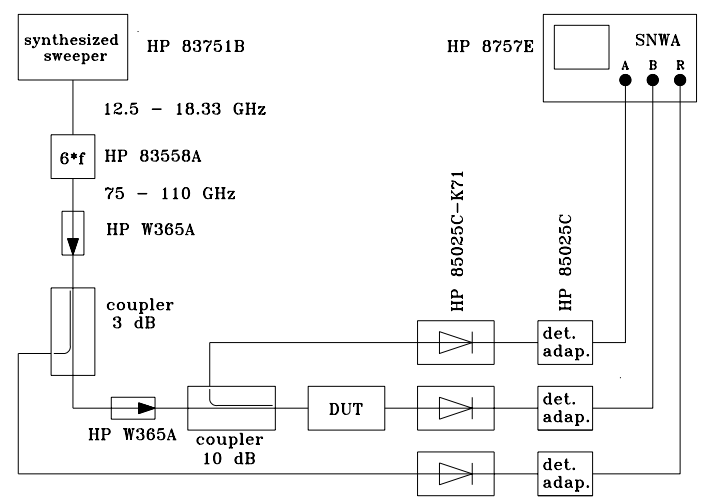

Figure 5. Block diagram for S11 and S12 measurements.

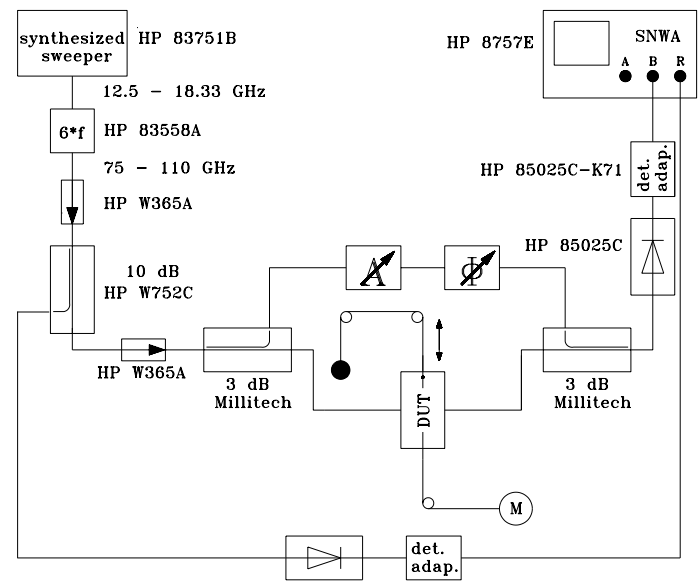

Figure 6. Block diagram for bead pull measurements.

The results of the transmission and reflection measurement are presented in figure 7 . The measured frequency response is in good qualitative agreement with the numerical simulation. The measured $4 \mathrm{~dB}$ loss in transmission is not yet understood. We believe that it has something to do with the surface roughness and the bad contact between the irises and the cavity. For bead pull measurements a nylon fiber with a diameter of ca. $0.05 \mathrm{~mm}$ and a knot as a bead was threaded through the beam pipe. The bead was pulled along the seven cells in longitudinal direction and the perturbed transmission coefficient at the input port was measured. The constant $\mathrm{c}$ in equation (1) depends on the shape of the knot and the input power level and is not determined at this time. Therefore, no unit is assigned to the field in figure 8 . Figure 8 shows the result of the measured amplitude of the $2 \pi / 3-$ mode at $91.392 \mathrm{GHz}$, determined with bead pull.

$$
\Gamma_{p}(\mathrm{z})=c|\mathrm{E}(\mathrm{z})| e^{-j \Phi(\mathrm{z})}
$$

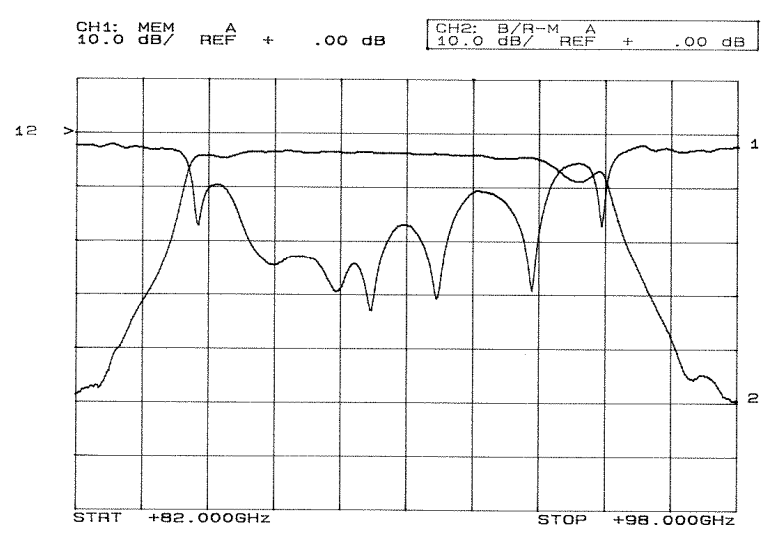

Figure 7. Reflection and transmission coefficient, measurement.

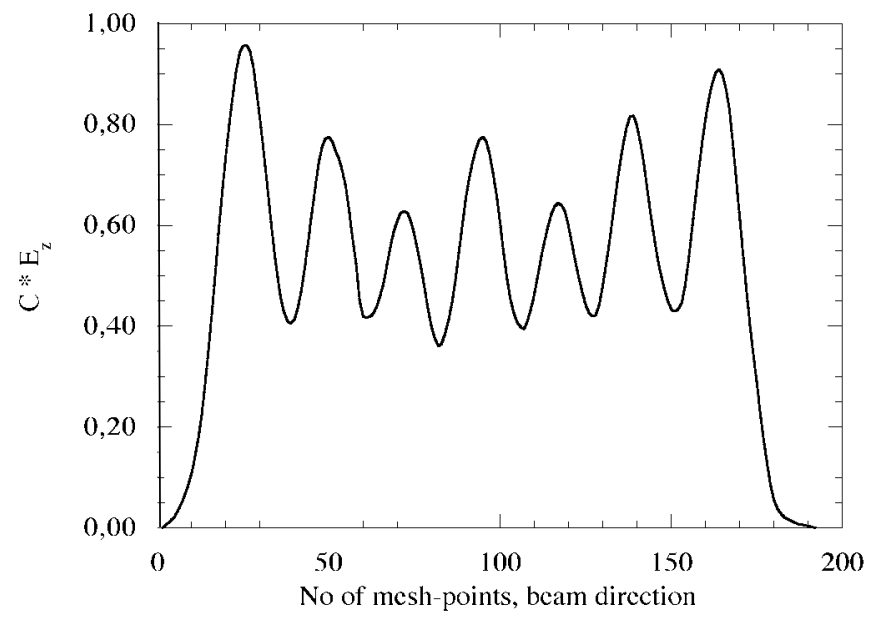

Figure 8. Field distribution measured with bead pull.

\section{ACKNOWLEDGMENT}

Part of the work was done at SLAC by one of the authors (R. Merte). We would like to thank the SLAC staff for their generous help and support.

\section{REFERENCES}

[1] H. Henke, Y.W. Kang and R.L. Kustom, A mm-wave RF Structure for Relativistic Electron Acceleration, Argonne National Laboratory, internal report ANL/APS/MMW-1, 1993.

[2] R. Merte, First Design of a W-Band Muffin Tin, Cold Test Model, Internal Note, Inst. f. Theoretische Elektrotechnik, TUBerlin.

[3] W. Bruns, GdfidL: A finite difference program for arbitrarily small perturbations in rectangular geometries, IEEE Trans. Magn. Vol. 32, No. 3 May 1996.

[4] P.J. Chou, R.H. Siemann, et. al., The Fabrication of Millimeter-Wavelength Accelerating Structures, SLAC-PUP-7339, November 1996. 